CARTAS AL EDITOR

Rev Chil Salud Pública 2019,

Vol 23(1): 81-82

\title{
SISTEMA DE VIGILANCIA EPIDEMIOLÓGICA DE PATOLOGÍAS BUCALES A DIEZ AÑOS DE DISTANCIA
}

TEN YEARS OF THE EPIDEMIOLOGICAL SURVEILLANCE SYSTEM FOR ORAL PATHOLOGIES

Estimado editor:

Una de las funciones esenciales de la Salud Pública es la evaluación y análisis del estado de salud de la población; por consiguiiente, es una obligación de las instituciones que conforman los servicios de salud del país obtener información al respecto. Uno de los instrumentos más utilizados en salud bucal es la información recabada por los índices de salud bucal (higiene oral, gingivitis, caries, etc.). En México se creó la Dirección General de Epidemiología y el Programa de Salud Bucal que generó un sistema para recopilar información oportuna y permanente del estado de salud bucal de la población mexicana. A partir del año 2009, ya con las 32 entidades federativas operando en 355 unidades centinela, se decidió realizar además de boletines trimestrales, una publicación anual de los resultados del Sistema de Vigilancia Epidemiológica de Patologías Bucales (SIVEPAB). Dicha información de fase permanente corresponde únicamente a las consultas de tratamiento dental en los consultorios de los Centros de Salud o unidades médicas del IMSS-Prospera, ISSSTE, SEDENA, SEMAR, PEMEX, DIF, los Servicios de Salud Estatales pertenecientes a la Secretaria de Salud, y de la Universidad de Guadalajara. Para procesar los datos se construyó un sistema informático a cargo del Centro Nacional de Programas Preventivos y Control de Enfermedades (CENAPRECE, antes CENAVECE) y para el año 2006 se crea el módulo de captura en la plataforma única de la Dirección General de Epidemiología, con lo cual integra al SIVEPAB como un sistema especial dentro del Sistema Nacional de Vigilancia Epidemiológica (SINAVE), para análisis de información y se unificaron las bases de datos de ambas plataformas. Para el desarrollo de actividades de vigilancia epidemiológica, el Comité Nacional de Salud Bucal las incluyó dentro de la Norma Oficial Mexicana NOM-013-SSA2-20061, en el numeral 10, propone registro y notificación epidemiológica. En conclusión, a diez años de distancia del establecimiento el SIVEPAB ha demostrado ser un sistema de información adecuado para conocer la salud bucal de la población mexicana, desde su instalación el año 2009 en todas las entidades del país.

Conflicto de intereses: No existe 


\section{REFERENCIAS BIBLIOGRÁFICAS:}

1. Secretaría de Salud, Subsecretaría de Prevención y Promoción de la Salud. Resultados del Sistema de Vigilancia Epidemiológica de Patologías Bucales (SIVEPAB) 2012. Centro Nacional de Programas Preventivos y Control de Enfermedades. México, Distrito Federal.

2. Boletín del Sistema de Vigilancia Epidemiológica de Patologías Bucales (2012). Secretaría de Salud. Dirección General de Epidemiología.

3. Estructura Orgánica Básica con base en el Decreto que reforma, adiciona y deroga diversas disposiciones del Reglamento Interior de la Secretaría de Salud. Diario Oficial de la Federación 02/02/2010.

4. SSA, CENAPRECE, Programa de Salud Bucal. Encuesta de Caries Dental en Escolares 1999-2001.

5. Dirección General de Información en Salud. Secretaría de Salud. Cubos dinámicos. Proyecciones de la Población de México 1990 - 2030. CONAPO. Consejo Nacional de Población. Proyecciones de la Población 2010-2050. Disponibles en http://www.dgis.salud. gob.mx/contenidos/basesdedatos/bdc_poblacion. $\mathrm{html}$ 